\title{
Volatility Reducing Effect by Introducing a Price Stabilization Agent on Cryptocurrencies Trading
}

\author{
Kyohei Shibano \\ Dept. of Technology Management for \\ Innovation \\ School of Engineering \\ The University of Tokyo, Japan \\ shibano@tmi.t.u-tokyo.ac.jp
}

\author{
Ruxin Lin \\ Dept. of Technology Management for \\ Innovation \\ School of Engineering \\ The University of Tokyo, Japan \\ linruxin@g.ecc.u-tokyo.ac.jp
}

\author{
Gento Mogi \\ Dept. of Technology Management for \\ Innovation \\ School of Engineering \\ The University of Tokyo, Japan \\ mogi@tmi.t.u-tokyo.ac.jp
}

\begin{abstract}
Cases of introducing token economy in designs of ICT services are increasing. Users in the early stages of the service are expected to participate in and be active in the service by expecting future price increases in that cryptocurrency. However, the volatility of cryptocurrencies is always intense, and the large volatility may cause users to be more interested in price changes than service activities, which diminishes the incentives for the service activities. In this study, in order to dampen the volatility of cryptocurrencies at the initial stage of their service launch, we assume the case where the service providers make bids to suppress the price changes based on the funds obtained from ICO, and conduct analysis using simulations in artificial market. In order to reproduce the actual price movement in the artificial market, we built an agent model that has the same stylized facts as the price movement of newly listed cryptocurrencies. Then, we introduced a price stabilization agent, and obtained a parameter set that reduces price volatility while suppressing the change in the slope of a simple linear regression compared to the original state using an optimization method. As a result, by introducing the price stabilization agent, we found a parameter set that can reduce the standard division of percentage changes by about $14 \%$ from the original price movement, and keep the slope of the simple linear regression trend at a $3.5 \%$ change.
\end{abstract}

\section{CCS Concepts}

- Computing methodologies $\rightarrow$ Agent / discrete models

\section{Keywords}

Cryptocurrency; Volatility; Blockchain; Token Economy.

\section{INTRODUCTION}

Token economy is one of the applications of blockchain. This is designed based on incentives for cryptocurrency (token) exchanges between players at the service assumed by the project and is realized using original cryptocurrencies and the smart contracts implemented on blockchain. Participating in the service in anticipation of a rise in cryptocurrency price, or taking some actions to boost the service for the rise in price, are some methods

\footnotetext{
Permission to make digital or hard copies of part or all of this work for personal or classroom use is granted without fee provided that copies are not made or distributed for profit or commercial advantage and that copies bear this notice and the full citation on the first page. Copyrights for third-party components of this work must be honored. For all other uses, contact the Owner/Author.

ICBCT'20, March 12-14, 2020, Hilo, HI, USA

(C) 2020 Copyright is held by the owner/author(s).

ACM ISBN 978-1-4503-7767-6/20/03.

https://doi.org/10.1145/3390566.3391679
}

to incent users in the early stage of a service. The incentive for users to use one service in the phase where the number of users is extremely small is brought about by the rising expectation of the cryptocurrency price. Nevertheless, cryptocurrencies have very large price volatility at exchanges. Even some major cryptocurrencies like Bitcoin or Ethereum have greater volatility compared to price movements of stocks and foreign exchange, let alone cryptocurrencies with few traders in their early stage, which tend to be more volatile. If the price volatility is too large, instead of focusing their efforts on service activities, users will pay more attention on selling cryptocurrencies at the moment of price increases at the exchange. This means that the incentive to perform service activities based on expectations of future price increases will disappear. In this study, in order to dampen the volatility of cryptocurrencies, which at their initial stage of service, some analyses will be made concerning placing bids on exchanges when price volatility is becoming higher using the money obtained from ICO (Initial Coin Offering) as capital, by the method of artificial market.

\section{PREVIOUS STUDIES}

\subsection{Token Bonding Curves (TBC)}

Token Bonding Curves concept is first proposed by Simon de la Rouviere in his posts about curation market, elaborates a mechanism called smart contract that formulating the price rules of tokens of a given type for exchange with another token [1]. For the price rule has been set in advance, so the token bonding curves can be expected to play a role in dampening volatility of the price in short time. But Simon also point out that when trading of another token is more valuable, there are more incentives to keep the token liquid, rather than bonding it, thus the volatility of price will happen.

Also, token bonding curve makes the price of a token increases as the supply increases, which can incentive the early contributors to act more actively. This concept is close to the token economy this paper wants to build. Although the volatilities of tokens which are using Token Bonding Curves are still large, the improved effect of the adoption of some other methods can be expected.

\subsection{Stablecoins}

Another effort trying to reduce the volatility of cryptocurrency is stablecoins---a price stable cryptocurrency whose market price is pegged to another stable asset to maintain stable exchange rates [2]. Seigniorage Shares is one of the approaches of stablecoins, which increases or decreases the amount of coin issued to control supply and demand, and then keeps the exchange rate constant [3]. This mechanism is kind of like the currency intervention. But what stablecoins pursue is not so much the reduction of volatility as the maintenance of a stable exchange rate, meaning that the 
value of stablecoins will not rise in the future, which is different from the purpose of this paper.

\subsection{Non- cryptocurrency Methods}

When facing extreme volatility, policy makers of stock market propose circuit breakers to curb traders [4]. Circuit breakers are trading rules that limit trading activity which include methods like trading halts, price limits, trading collars and so on.

In addition, in the event of a sudden change in the exchange rate of a currency, a government or a central bank intervenes in the foreign exchange market by buying or selling foreign currency in exchange for their own domestic currency for the purpose of keeping stability, which is known as currency intervention [5]. The method of currency intervention is quite familiar with the method of this paper, so what if there is one playing the role of the government or the central bank in cryptocurrency market? We will do some simulations to find the answer.

\section{METHODOLOGY}

In this study, we use the artificial market simulator Plham [6, 7, 8]. Changes in price volatility when a price stabilization agent is assumed are analyzed by simulation using Plham.

\subsection{Artificial Market}

Artificial market is a social simulation method that derives price movements from two micro factors of investor behavior and pricing mechanism at the exchanges. Artificial market is used for analyses of various financial markets such as stock markets and foreign exchange markets $[9,10,11,12]$. There are also examples used for analysis to cryptocurrency markets [13].

The statistical properties of macro quantities are called stylized facts [14]. Examples of stylized fact include fat-tail and volatilityclustering. Fat-tail is a phenomenon in which the kurtosis of the distribution of the percentage change in market prices is predominantly positive. Volatility-clustering is a phenomenon where volatility depends on time and tends to continue as volatility increases [14, 15, 16]. Many other stylized facts have also been discovered, but their existence is not as stable as those two. Fat-tail and volatility-clustering are known to exist prevailing regardless of assets, regions or time, and exist universally to same extent $[14,17]$. In this study, the fat-tail index is the kurtosis of percentage change, while the volatility-clustering index is the autocorrelation coefficient for square percentage change [17].

Plham is a software for conducting artificial market simulation and is open source $[6,7,8]$. Various agents and markets have already been implemented. In addition to being able to use the existing agents and markets, it is designed to easily implement new agents by oneself.

\subsection{Design of the Simulation Analysis}

First, the design of artificial market in this study will be introduced. Concerning the agent which perform the investment behavior, FCNAgent implemented in Plham which performs basic investment behavior is used. FCNAgent is based on [18] to calculate expected return and expected price using the weighted average of Fundamental Analysis (F), Chart Analysis (C) and Regular Noise $(\mathrm{N})$. And then, it compares the expected price in the future with the current price and place a buy/sell order according to their magnitude relationship. FCNAgent has four characteristics [16] of that almost $100 \%$ sell or buy orders will be placed, that the transaction volume is always being only 1 unit, that usually only trades one stock, that the expected return does not depend on the amount of assets held by the agent.

Next, the market we used is "Market" implemented in Plham: continuous double auction. Market stores time series of prices up to the present time including time $t$. The order placed by the agent at time $t$ determines the price at time $t+1$. Under the circumstances that multiple orders are executed between some time $t$ and $t+1$, the price of the last transaction will become the market price at time $t+1$. After the price update process above, the order at the next time $t+1$ will be accepted. Each of the all agents calculates the predicted value of the price in period $t+1$ based on the price in period $t$. And based on that, they determine investment behaviors and place orders. The orders placed are executed in continuous double auction method and the price of period $t+1$ will be determined. For details of FCNAgent and Market, see [16].

\subsection{FCNAgent Parameter Settings}

FCNAgent has some parameters and that parameter set of FCNAgent is determined by the following methods. Price movement in artificial market simulation is determined by the agent, and in this case, it is determined by parameters in FCNAgent.In order to reproduce the same price movement as initial listing of cryptocurrencies, the actual price data of cryptocurrencies in their initial listing period is used, to obtain the parameters of FCNAgent which make its stylized facts equivalent to the stylized facts derived from that data. About ten cryptocurrencies that were conducted ICO between 2017 and 2019 are randomly extracted every quarter, and the price data is obtained from CoinMarketCap [19]. However, each value of stylized facts varies greatly for each cryptocurrency. This time, for each cryptocurrency, extracting the data of 35 days from the first day of listing, and then 8th to 42rd days, 15th to 49th days, ..., 57th to 91st days, with the width shifted by 7 days. Then a total of 693 stylized facts for 77 cryptocurrencies with 9 groups of 35-day data each are calculated, and the range of values most likely to appear is adopted. Regarding the range of values, 0.5 is used for the kurtosis of percentage changes (fat-tail) and 0.025 is used for the autocorrelation coefficient for square percentage changes (volatility-clustering). Table 1 shows the basic statistics of the 35-day cryptocurrency price data after listing that being used. And table 2 shows the values of stylized facts. ${ }^{1}$

Table 1. Basic information of cryptocurrency for 35 days

\begin{tabular}{|l|r|}
\hline Data Acquisition Frequency & Closing price for a day \\
\hline Data Source & CoinMarketCap [19] \\
\hline Number of Target Cryptocurrencies & 77 \\
\hline ICO Period & $2017 / 01-2019 / 06$ \\
\hline Average of Percentage Changes ${ }^{2}$ & 0.00790 \\
\hline SD of Percentage Changes ${ }^{2}$ & 0.187 \\
\hline
\end{tabular}

\footnotetext{
${ }^{1}$ The histogram of fat-tail and volatility-clustering is shown in Appendix Figure 2.

2 The average and standard deviation values of percentage changes here are calculated from a total of 6,930 data of 90 days $\times 77$ cryptocurrencies excluding the first day from the 91-day data used in the calculation of stylized facts.
} 
Table 2. Stylized facts of cryptocurrency for 35 days

\begin{tabular}{|c|c|c|c|c|}
\hline & mean & SD & Range of Mode \\
\hline \multicolumn{2}{|c|}{$\begin{array}{lr}\text { Kurtosis of } \\
\text { Percentage Changes }\end{array}$} & 3.16 & 5.14 & {$[0,0.5]$} \\
\hline \multirow{6}{*}{$\begin{array}{l}\text { Auto- } \\
\text { correlation } \\
\text { coefficient } \\
\text { for Square } \\
\text { Percentage } \\
\text { Changes }\end{array}$} & Lag 1 & 0.0704 & 0.158 & {$[-0.05,-0.025]$} \\
\hline & Lag 2 & 0.00834 & 0.152 & {$[-0.05,-0.025]$} \\
\hline & Lag 3 & 0.00888 & 0.139 & {$[-0.05,-0.025]$} \\
\hline & Lag 4 & 0.00800 & 0.142 & {$[-0.05,-0.025]$} \\
\hline & Lag 5 & -0.0208 & 0.119 & {$[-0.05,-0.025]$} \\
\hline & Lag 6 & -0.0268 & 0.124 & {$[-0.05,-0.025]$} \\
\hline
\end{tabular}

In this simulation, assuming each $t$ is 1 minute, $t=50400$, and conduct the simulation over a period of 35 days. The price per day $(t=1440)$ is adopted as the closing price of each day, and the stylized facts are calculated for 35-day price changes. In addition, in artificial market simulations, even if the same parameters are assumed, the price changes will differ from trial to trial of the simulation. Therefore, we execute the simulations 100 times and calculate stylized facts of each simulation. Then, the average value is used as the stylized fact of the parameter set. Let the kurtosis of percentage changes be $f t$, the autocorrelation coefficient for square percentage changes be $v c_{i}$ for Lag $i$ ( $i=$ $1, \ldots, 6)$. The range of the stylized fact of the cryptocurrency in its initial listing period obtained above is $F T^{M O D E}$ for fat-tail and $V C_{i}^{M O D E}$ for volatility-clustering for each $i$. At this time, the distance diff from the stylized fact of the cryptocurrency in its initial listing period is

$$
\operatorname{diff}=\alpha \mathrm{d}\left(\mathrm{ft}, F T^{M O D E}\right)+\frac{1}{6} \sum_{i=1}^{6} d\left(v c_{i}, V C_{i}^{M O D E}\right)
$$

for any stylized facts $f t$ and $v c_{i}$, and the FCNAgent parameter set is obtained by seeking the parameters that make diff smaller ${ }^{3}$. scipy.optimize.minimize [20] in Python is used as optimization tools. Table 3 shows the parameters of FCNAgent used in the simulation of this study, including the values obtained as a result of seeking.

Table 3. Parameters of FCNAgent

\begin{tabular}{|c|c|c|}
\hline fundamental_weight & $\begin{array}{c}\text { Random numbers } \\
\text { following exponential } \\
\text { distribution, } \\
\lambda=0.5077\end{array}$ & $\begin{array}{c}\lambda \text { is obtained } \\
\text { by seeking }\end{array}$ \\
\hline chart_weight & $\begin{array}{c}\text { Random numbers } \\
\text { following exponential } \\
\text { distribution, } \\
\lambda=0.2\end{array}$ & $\begin{array}{c}\lambda \text { is obtained } \\
\text { by seeking }\end{array}$ \\
\hline noise_weight & $\begin{array}{c}\text { Random numbers } \\
\text { following exponential } \\
\text { distribution, } \\
\lambda=0.5\end{array}$ & $\begin{array}{c}\text { Assuming a } \\
\text { fixed value }\end{array}$ \\
\hline noise_scale & 0.0000938 & $\begin{array}{c}\text { Obtained by } \\
\text { seeking }\end{array}$ \\
\hline
\end{tabular}

${ }^{3} d(\cdot, \cdot)$ is the distance between points and the set, and $\alpha$ is the value of maximum absolute value of the range in all $i$ of $V C_{i}^{M O D E}$ divides by the maximum absolute value of the values in $F T^{M O D E}$. Since the value of fat-tail tends to be larger than the value of volatility-clustering, $\alpha$ is multiplied.

\begin{tabular}{|c|c|c|}
\hline time_window_size & $\begin{array}{c}\text { Uniform random number } \\
\text { in the interval }[2,12]\end{array}$ & $\begin{array}{c}\text { Obtained by } \\
\text { seeking }\end{array}$ \\
\hline order_margin & $\begin{array}{c}\text { Uniform random number } \\
\text { in the interval [0.00813, } \\
0.108]\end{array}$ & $\begin{array}{c}\text { Obtained by } \\
\text { seeking }\end{array}$ \\
\hline margin_type & fixed & $\begin{array}{c}\text { Assuming a } \\
\text { fixed value }\end{array}$ \\
\hline iteration_steps & 50400 & $\begin{array}{c}\text { Assuming a } \\
\text { fixed value }\end{array}$ \\
\hline market_price & 300 & $\begin{array}{c}\text { Assuming a } \\
\text { fixed value }\end{array}$ \\
\hline tick_size & 0.00001 & $\begin{array}{c}\text { Assuming a } \\
\text { fixed value }\end{array}$ \\
\hline num_agents & 100 & $\begin{array}{c}\text { Assuming a } \\
\text { fixed value }\end{array}$ \\
\hline outstanding_shares & 250000000 & $\begin{array}{c}\text { Assuming a } \\
\text { fixed value }\end{array}$ \\
\hline
\end{tabular}

\subsection{Introduction of a Price Stabilization Agent}

In order to reduce the price volatility, we introduce the price adjustment agent: StablilizationAgent. This agent is supposed to simulate the service operator of the target cryptocurrency who issued the cryptocurrency and place bids on funds raised by ICO to reduce the volatility. If the average value of the daily price changes in the most recent interval period exceeds the threshold: threshold, sell the bids at the price before interval period and bidding each time in a volume of bidding_volume. On the contrary, if the price change is below -threshold, the buying bids are placed, and the bidding volume is set by bidding_volume. The bidding_volume at this time is sufficiently large, and if bidding is not executed, bidding will continue in bidding_time_lenghth period. The settings of FCNAgent are exactly the same as in Table 3, and how price changes when one agent of StabilizationAgent is introduced and is not introduced will be analyzed. The role of Stabilization Agent is to suppress price volatility without affecting med-to-long term price trend. Here in this paper, we calculated the simple linear regression, and use the slope of it as a mid-to-long term price trend.

For the four parameters above, use scipy.optimization.minimize [20] as in the case of FCNAgent to find a parameter set that make the sum of percentage changes' variance value and simple linear regression's slope smaller. The result is shown in Table 4.

Table 4. Parameters of StabilizationAgent

\begin{tabular}{|c|c|}
\hline bidding_volume & 10000 \\
\hline bidding_time_length & 62 \\
\hline interval & 76 \\
\hline threshold & 0.0995 \\
\hline
\end{tabular}

\subsection{Simulation Results}

The movements of percentage changes with and without StabilizationAgent with the parameters in Table 4 are shown in Figure 1.

Figure 1 shows the movement of the percentage changes under 100 trials of simulation and the left side is the one with StabilizationAgent while the right side is without StabilizationAgent. It can be seen that the percentage changes are slightly close to zero overall. Table 5 summarizes the means of slope of simple linear regression lines and standard deviations of the percentage changes in 100 trials in this case. These are the 
values for the total percentage changes of 3500, which was simulated 100 times for 35 days.

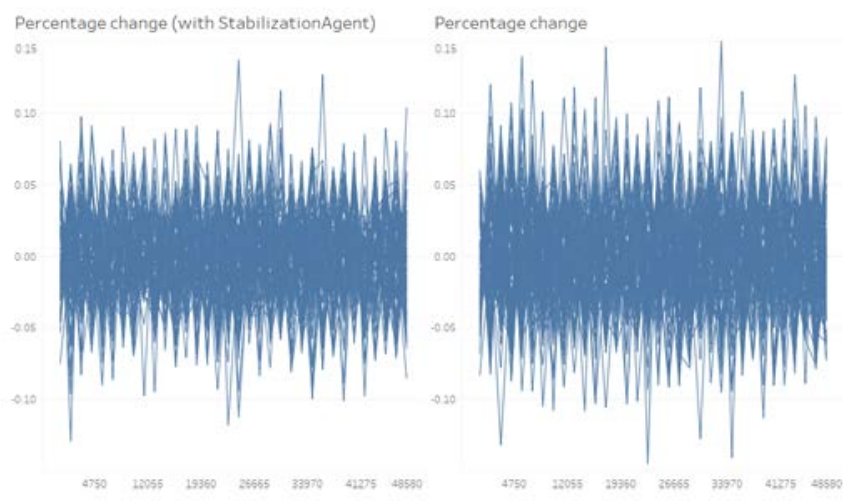

Figure 1. Movements of percentage changes with and without StabilizationAgent

Table 5. Changes in percentage change depending on whether StabilizationAgent is introduced

\begin{tabular}{|l|l|c|}
\hline $\begin{array}{l}\text { Without } \\
\text { StabilizationAgent }\end{array}$ & $\begin{array}{l}\text { Mean slope of simple } \\
\text { linear regression lines }\end{array}$ & -0.0484 \\
\hline $\begin{array}{l}\text { Without } \\
\text { StabilizationAgent }\end{array}$ & SD of percentage change & 0.0364 \\
\hline $\begin{array}{l}\text { With } \\
\text { StabilizationAgent }\end{array}$ & $\begin{array}{l}\text { Mean slope of simple } \\
\text { linear regression lines }\end{array}$ & -0.0467 \\
\hline $\begin{array}{l}\text { With } \\
\text { StabilizationAgent }\end{array}$ & SD of percentage change & 0.0313 \\
\hline
\end{tabular}

When the Levene test was performed on the percentage change in these two cases, the p-value was 3.12e-13, and it was confirmed that there was a significant difference of SD at $p<0.05$. At this time, the SD of the percentage changes could be reduced by about $14 \%$. On the other hand, the slope of the simple linear regression before and after the introduction of StabilizationAgent is changed from -0.0484 to -0.0467 so that it changes only about $3.5 \%$.

\subsection{Discussion}

It was found that the price volatility can be suppressed by introducing StabilizationAgent.

The purpose of this study is to confirm how much the price volatility can be dampened by making bids by service providers with the funds obtained from ICO for cryptocurrencies in their initial listing period. What is important is to realize that the return is left as it is while the volatility is dampened. If the service providers conduct price intervention that greatly varies the return, even the volatility is dampened, the credit of the cryptocurrency will lose, and the users will leave. From the results of simulations, we can see that SD was able to be suppressed with respect to percentage change, and that the change of the mean slope of the simple linear regression lines at that time was also within the range of 3.5\%, which didn't make the return change greatly.

In the simulations, the fundamental price is fixed. Although it is difficult to find a fundamental price for each cryptocurrency, for the news about the services related to the cryptocurrency can be a big influence factor. We would like to follow up on changes in fundamental prices and continue to study whether the volatility can be dampened only by the activities of StabilizationAgent.
On the other hand, the method in this study is only an analysis by simulations. In the artificial market, we tried to reproduce the price changes with the activities of FCNAgent in a close state to the stylized facts that can reproduce the price changes of a cryptocurrency in its initial listing period. But as a practical issue of a cryptocurrency in your own project, further research will be needed to determine whether the obtained StabilizationAgent parameter set can effectively dampen the volatility. It may be good to refer the past cryptocurrency that has similar qualitative characteristics to the project, and then reproduce the price changes close to the stylized facts of the cryptocurrency and obtain the parameters of StabilizationAgent.

Considering the practical implementation of StabilizationAgent, it is difficult to bid directly from the Smart Contract to exchanges. The service providers hold the exchange account and use that account to realize StabilizationAgent with funds raised by ICO. In that case, it will be required to disclose the bidding history and balance information, but the problem remains whether it can be realized by trustless.

\section{CONCLUTION}

In this study, the parameter set of FCNAgent that reproduces the price changes of the cryptocurrency in its initial listing period using artificial market was obtained, and the change in percentage changes with and without the introduction of StabilizationAgent is calculated. As a result, it was confirmed that the percentage change can be reduced by introducing StabilizationAgent.

\section{APPENDIX}

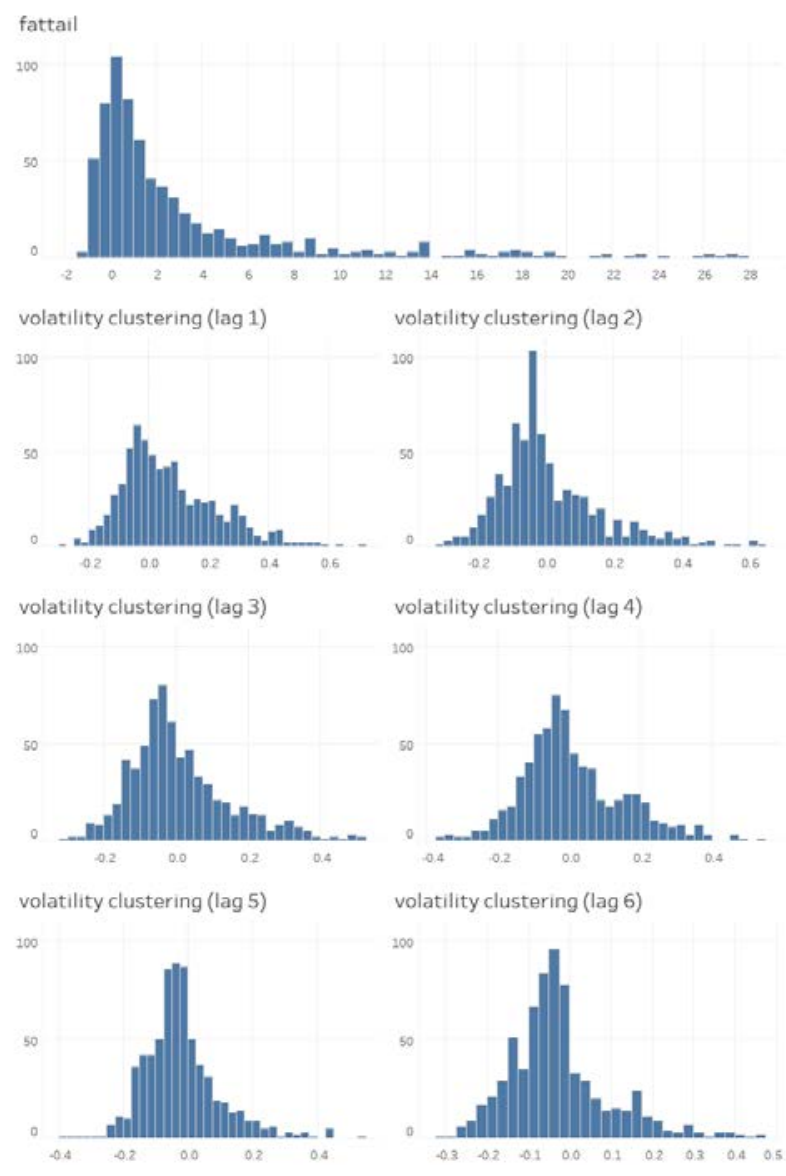

Figure 2. Histogram of actual data stylized facts 


\section{REFERENCES}

[1] Simon de la Rouviere. 2017. Curation Market. https://docs.google.com/document/d/1VNkBjjGhcZUV9CyC 0ccWYbqeOoVKT2maqX0rK3yXB20/edit (accessed 201911-6).

[2] Ingolf G.A. Pernice, Sebastian Henningsen, Roman Proskalovich, Martin Florian, Hermann Elendner, Bjo ïn Scheuermann. 2019. Monetary Stabilization in Cryptocurrencies --- Design Approaches and Open Questions.

[3] Robert Sams 2015. A Note on Cryptocurrency Stabilization: Seigniorage Shares

[4] Larry Harris 2003. Trading and Exchanges: Market Microstructure for Practitioners. Oxford University Press. pp. 572-578

[5] K. Tsujimura, M. Mizoshita. 2003. Funds Circulation Analysis on Effects of Currency Intervention and Sterilization Policy, 11(2), PAPAIOS, 49-62, DOI=https://doi.org/10.11107/papaios.11.49, in Japanese

[6] Torii, T., Kamada, T., Izumi, K., \& Yamada, K. (2017). Platform Design for Large-scale Artificial Market Simulation and Preliminary Evaluation on the K Computer. Artificial Life and Robotics, 22(3), 301-307.

[7] Plham documentation, https://plham.github.io/ (accessed 2019-12-04), in Japanese

[8] Plham project repository, https://github.com/plham (accessed 2019-12-04)

[9] Kiyoshi Izumi. 2003. Artificial Market: Complexity Approach to Market Analysis. Morikita Publishing Co., in Japanese

[10] LeBaron, B. 2006. Agent-based Computational Finance, Handbook of computational economics, Vol.2, pp.1187-1233
[11] Chen, S.-H., Cang, C.-L., and Du, Y.-R. 2012. Agent-based Economic Models and Econometrics, Knowledge Engineering Review, Vol. 27, No. 2, pp.187-219

[12] Cristelli, M. 2014. Complexity in Financial Markets, Modeling Psychological Behaviour in Agent-Based Models and Order Book Models, Springer

[13] Cocco, L., Concas, G., and Marchesi, M. 2017. Using an Artificial Financial Market for Studying a Cryptocurrency Market, Journal of Economic Interaction and Coordination, Vol. 12, Issue 2, pp 345-365.

[14] Sewell, M. 2011. Characterization of Financial Time Series, RN, 11(01)

[15] Mandelbrot, B. 1972. Statistical methodology for nonperiodic cycles: from the covariance to $R / S$ analysis. In Annals of Economic and Social Measurement, Volume 1, Number 3 (pp. 259-290). NBER.

[16] Cont, R. 2001. Empirical Properties of Asset Returns: Stylized Facts and Statistical Issues. QUANTITATIVE FINANCE, 1, 223-236.

[17] Takanobu Mizuta. 2014. Analysis of Financial Market Regulations and Systems Using Artificial Market Simulations. Doctoral dissertation, University of Tokyo., in Japanese

[18] Chiarella, C., \& Iori, G. (2002). A Simulation Analysis of the Microstructure of Double Auction Markets*. Quantitative finance, 2(5), 346-353.

[19] CoinMarketCap, https://coinmarketcap.com/ (accessed 2019-12-04)

[20] scipy.optimize.minimize, https://docs.scipy.org/doc/scipy/ reference/generated/scipy.optimize.minimize.html (accessed 2019-12-04) 